\title{
Grundwasser - die Qualität muss stimmen!
}

\author{
Nico Goldscheider ${ }^{1}$ (D) Christoph Neukum $^{2}$
}

Eingegangen: 24. November 2017 / Online publiziert: 22. Dezember 2017 (C) Springer-Verlag GmbH Deutschland, ein Teil von Springer Nature 2017

Ab Januar 2018 übernehmen wir für vier Jahre die Aufgabe als Editoren der Zeitschrift Grundwasser, gemeinsam mit einem Team von 24 Assoziierten Editoren, darunter 18 neue Gesichter und 6 Kollegen, die bereits in den vergangenen Jahren mitgearbeitet haben, also eine Mischung aus ,frischem Wind“ und „Kontinuität“. Neben Hydrogeologen aus Deutschland, Österreich und der Schweiz, sind auch Kollegen aus Kanada, Slowenien, Ungarn und dem Libanon mit dabei. Bei Gudrun Massmann und Traugott Scheytt bedanken wir uns für ihre gute Arbeit in den vergangenen vier Jahren und für die reibungslose und kollegiale Übergabe.

In den kommenden Jahren sieht sich die Hydrogeologie in Deutschland, Österreich und der Schweiz, aber auch international, großen und vielfältigen Herausforderungen gegenüber.

Dazu gehört sicherlich der nächste Bericht zur Bestandaufnahme der EU-Wasserrahmenrichtlinie 2019. Viele Wasserversorger kämpfen mit hohen und teils steigenden Nährstoffkonzentrationen im Grundwasser. Der Nitrat-Grenzwert von $50 \mathrm{mg} / \mathrm{l}$ ist in vielen Regionen überschritten. Eine bedeutende Nitrat-Quelle ist hierbei die Landwirtschaft, die mit der Ausbringung von Nährstoffen über den tatsächlichen Pflanzenbedarf hinaus zur Verschlechterung der Grundwasserqualität beiträgt. Ein weiteres hochbrisantes,

Nico Goldscheider

goldscheider@kit.edu

Christoph Neukum

christoph.neukum@bgr.de

1 Inst. f. Angew. Geowissenschaften, Karlsruher Institut für Technologie, Kaiserstr. 12, 76131 Karlsruhe, Deutschland

2 Bundesanstalt für Geowissenschaften und Rohstoffe, B2.3 Grundwasserressourcen, Beschaffenheit und Rohstoffe, Stilleweg 2, 30655 Hannover, Deutschland wenn auch regional begrenztes Problem, ist die Kontamination des Grundwassers mit per- und polyfluorierten Chemikalien (PFC). Diese und andere Fragen rund um die Wasserqualität werden sicher auch in dieser Zeitschrift zunehmende Aufmerksamkeit erfahren.

Nicht nur für die Trinkwasserversorgung muss die Grundwasserqualität stimmen, sondern auch für alle Ökosysteme, die direkt oder indirekt vom Grundwasser abhängig sind. Hydrogeologie und Ökologie sollten daher in Zukunft viel stärker Hand in Hand gehen. Hier gibt es viele gemeinsame Interessen, aber auch Konfliktfelder. Einerseits haben sowohl der Umwelt- und Naturschutz, als auch die Wasserversorger Interesse an einer möglichst sauberen und intakten Natur. Andererseits gibt es aber auch Interessenkonflikte, da Grundwasserabsenkungen empfindliche Ökosysteme beeinträchtigen können und so manches Wasserschutzgebiet zumindest teilweise auch Natura2000Gebiet ist. Ziel sollte daher ein ökologisches Grundwassermanagement sein. Hier gibt es noch viel Potenzial für spannende Forschung und deren Umsetzung in die Praxis.

Gerade im Gebirge sind die Folgen des globalen Wandels besonders ausgeprägt. Der rapide Gletscherschwund in den Alpen ist hierfür wohl das augenfälligste Beispiel. Die Folgen für die damit verbundenen Grundwasservorkommen und die Auswirkungen für die Praxis sind vielfältig, aber bisher nur unzureichend erforscht. Hier zeigt sich ein weiteres Themenfeld für Grundwasser, mit besonders hoher Relevanz für unsere Autoren und Leser aus Österreich und der Schweiz.

Mit dem globalen Wandel und dem Druck auf unsere Grundwasserressourcen durch konkurrierende Nutzungen wird auch die Weiterentwicklung von Methoden und Verfahren zur Grundwassererkundung und zur Prognose von Eingriffen in den natürlichen Grundwasserhaushalt in $\mathrm{Zu}$ kunft wichtig bleiben. Insbesondere der Einsatz von nume- 
rischen Verfahren in Festgesteinen und komplexen hydrogeologischen Gebieten wird aller Voraussicht nach weiter zunehmen und kann viel Stoff für potenzielle Beiträge in Grundwasser liefern.

Ein wichtiges Themengebiet, das sich augenblicklich neu entwickelt und voraussichtlich auch längerfristig Aktualität haben wird, ist die Suche nach einem geeigneten Endlager für wärmentwickelnde radioaktive Abfälle. Nach der Novellierung des Standortauswahlgesetzes Anfang 2017 und der Gründung der Aufsichtsbehörde und der Vorhabenträgergesellschaft werden die Arbeiten zur Standortsuche bald Fahrt aufnehmen und für Forschung und Praxis neue Aufgaben und Herausforderungen bereitstellen.

Die angesprochenen Themen haben für Deutschland und die Nachbarländer eine hohe Aktualität und Relevanz. Auch wenn Hydrogeologen weltweit vor ähnlichen Herausforderungen stehen, hat die Hydrogeologie immer auch eine regionale Komponente und findet nicht nur in der akademischen Forschung statt, sondern auch und vor allem in Ingenieurbüros, bei Wasserversorgern sowie in Ämtern und Behörden. In der hydrogeologischen Praxis sind vielfältige Herausforderungen durch die jeweiligen Akteure zu meistern. Daraus kann sich eine Stärke der Zeitschrift Grundwasser ergeben, da sie nicht nur Forschungsergebnisse für die Praxis präsentiert, sondern auch die Problemstellungen aus der Praxis in die Forschung trägt und entsprechende Impulse setzt. Daher ist auch weiterhin eine deutschsprachige Fachzeitschrift notwendig und wichtig, die ein Bindeglied zwischen Forschung und Praxis bildet. Eine Zeitschrift mit diesem Anspruch wird niemals einen sehr hohen Impactfaktor erreichen, aber das ist auch nicht so wichtig - die Qualität muss stimmen!

Dass dies die Leser von Grundwasser mehrheitlich genauso sehen, hat die Umfrage gezeigt. Gleichzeitig ist Grundwasser aber auch eine internationale Fachzeitschrift. Auch in Zukunft sollen ausgewählte Beiträge auf Englisch veröffentlicht werden, und auch das Team der Assoziierten Editoren aus sieben Ländern steht für eine weitere Internationalisierung. Dennoch ist und bleibt Grundwasser die Zeitschrift der Fachgesellschaft FH-DGGV und richtet sich daher primär an die deutschsprachige "Community“ - wie heißt das doch gleich auf Deutsch? Da sehen Sie, wie wichtig es ist, auch die eigene Sprache zu pflegen!

Wir freuen uns auf vier produktive Jahre mit den Lesern und Autoren von Grundwasser und mit dem gesamten Team, das diese Zeitschrift aktiv gestaltet und qualitativ weiterentwickelt. 